\title{
Índice cintura estatura y agregación de componentes cardiometabólicos en niños y adolescentes de Santiago
}

\author{
PILAR ARNAIZ ${ }^{1,2}$, ARNALDO MARÍN $^{a}$, FELIPE PINO $^{a}$, SALESA BARJA ${ }^{1}$, \\ MARLENE AGLONY ${ }^{1}$, CARLOS NAVARRETE ${ }^{3, b}$, MÓNICA ACEVEDO $^{2}$
}

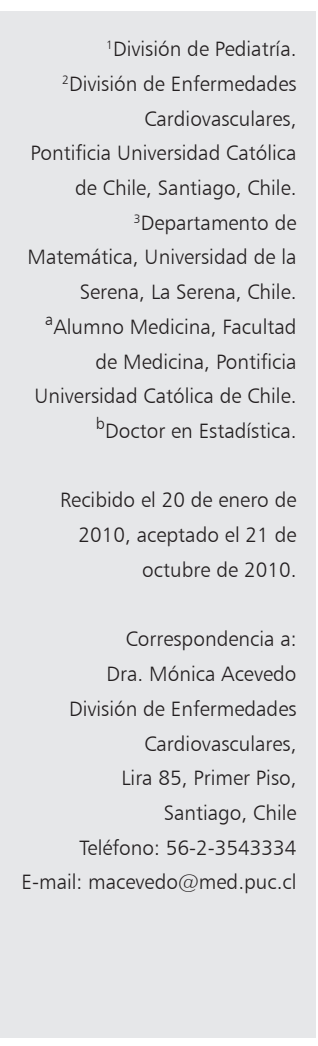

\section{Waist height ratio, ultrasensitive $\mathrm{c}$ reactive protein and metabolic syndrome in children}

Background: Waist to height ratio and ultrasensitive C-reactive protein are predictors of the presence of the metabolic syndrome in children. Aim: To determine the proportional risk of metabolic syndrome component clustering in children, using waist to height ratio and ultrasensitive C-reactive protein. Material and Methods: Anthropometric measures, blood pressure, fasting serum lipid profile, blood glucose and ultrasensitive $C$-reactive protein were determined in 209 children aged $11.5 \pm 2$ years (50\% females). The presence of the metabolic syndrome as a function of waist to height ratio and $C$-reactive protein was modeled using logistic regression equations. The risk of clustering one, two or more components of the metabolic syndrome was calculated. Results: Metabolic syndrome was present in 5\% of all children and 18\% of those that were obese. The cut off points for waist to hip ratio and ultrasensitive $C$-reactive protein were 0.55 and $0.61 \mathrm{mg} / \mathrm{L}$, respectively. For each 0.01 increment in waist to height ratio, the odds ratio of increasing one component of the metabolic syndrome was $1.2(1.15-1.25)$ or 15 to $25 \%$. The odds ratio for log-transformed ultrasensitive C-reactive protein was 1.62 (1.26-2.09). Excluding waist circumference, the odds ratio of adding one or more components of the metabolic syndrome was 1.05 (1.01-1.09) per 0.01 increment in waist to height ratio, but the odds ratio for $C$-reactive protein was no longer significant. Conclusions: Waist to height ratio and ultrasensitive $C$-reactive protein predict the risk of clustering components of the metabolic syndrome in these children.

(Rev Med Chile 2010; 138: 1378-1385).

Key words: C-Reactive protein; Metabolic syndrome X; Waist circumference.
$\mathrm{L}$ a epidemia de obesidad ha alcanzado también a los niños y jóvenes chilenos, con una prevalencia de $8 \%$ en menores de 6 años y $21 \%$ en escolares de primero básico ${ }^{1}$. El 35\% de los escolares entre 6 y 11 años tiene sobrepeso u obesidad según el Instituto Nacional de Estadística ${ }^{2}$, cifras que anticipan una alta prevalencia de síndrome metabólico (SMET) en los años venideros, aumentando así el riesgo de diabetes tipo 2 y enfermedad cardiovascular ${ }^{3-5}$. Datos reportados en
Estados Unidos de Norteamérica (EE.UU.) hablan de $4 \%$ a $9 \%$ de SMET en población general de adolescentes y $38 \%$ a $50 \%$ en adolescentes obesos ${ }^{6-8}$. En Chile se han reportado cifras similares ${ }^{9,10}$.

La mayoría de los investigadores actuales se basan en la definición de Cook y cols ${ }^{6}$ para determinar la presencia de SMET en niños y adolescentes. Sin embargo, esta definición basa el diagnóstico en la presencia de 3 o más componentes del SMET. De este modo, si un niño tiene sólo 2 componentes, 
no tendría SMET. La definición, por lo tanto, no alerta sobre la continuidad del riesgo metabólico, lo que es fundamental cuando hablamos de población pediátrica.

Numerosos parámetros antropométricos han sido utilizados para predecir el riesgo de SMET y diabetes. Varios estudios, en adultos y en niños, han mostrado un aumento en la prevalencia de SMET y sus componentes a medida que se incrementa el índice de masa corporal (IMC) (NHANES $)^{11,12}$. Trabajos epidemiológicos realizados en adultos han evaluado y validado la circunferencia de cintura (CC) como un importante predictor, no sólo de factores de riesgo cardiovascular, sino de diabetes mellitus tipo 2 y enfermedad coronaria $^{13-19}$. La CC también ha sido validada en niños como predictor de $\mathrm{SMET}^{20-23}$. La medición de IMC y CC en niños requiere comparar con percentiles según sexo y edad, lo que acarrea tiempos considerables.

Se ha planteado, entonces, que medidas antropométricas que incorporen la medición de la cintura y la forma corporal, como lo hace el índice cintura estatura (ICE), tendrían una mayor capacidad para predecir factores de riesgo relacionados con la obesidad en niños y adultos, reemplazando al IMC en las definiciones de diagnóstico clínico de $\mathrm{SMET}^{24-30}$. El ICE no requiere comparación en percentiles, siendo rápido y fácil de calcular en la práctica diaria. Un estudio en adultos chilenos mostró que el ICE es un predictor de mortalidad general más preciso que el IMC y que la razón cintura-cadera ${ }^{31}$.

Otro marcador de SMET y eventos cardiovasculares en adultos es la proteína $\mathrm{C}$ reactiva ultrasensible (PCRus), la que participa en el proceso aterogénico ${ }^{32,33}$. En niños, nuestro grupo recientemente publicó que la PCRus se asocia en forma directa y significativa a distintos parámetros antropométricos de adiposidad ${ }^{34}$.

Debido a la dificultad de expresar el real riesgo cardiometabólico en niños usando las definiciones tradicionales de SMET como la de Cook, el presente estudio plantea la hipótesis de que en nuestra población de niños y adolescentes, el ICE, y/o la PCRus, tendrían una buena capacidad predictiva para discriminar sobre la presencia de uno o más componentes del SMET. Para ello, determinamos el riesgo proporcional de agregación de dichos componentes, según ICE y PCRus, en una cohorte pediátrica.

\section{Método}

Estudio transversal en 209 escolares de estrato socioeconómico medio, medio bajo y bajo, pertenecientes a colegios municipales subvencionados del Santiago urbano, entre octubre de 2005 y diciembre de 2006. Los escolares fueron clasificados según el Índice de Desarrollo Urbano vigente $^{2}$. Además, se invitó a participar en forma voluntaria a niños que consultaron por primera vez por sobrepeso u obesidad en los policlínicos de la red de salud del Hospital Clínico de la Pontificia Universidad Católica de Chile durante el mismo período de tiempo y que fueran comparables en características socioeconómicas.

Los criterios de inclusión fueron edad entre 6 y 16 años, ausencia de antecedentes de trastornos del metabolismo glucídico, dislipidemias, hipertensión, enfermedades metabólicas, infecciones y terapia farmacológica reciente. El adulto responsable firmó un consentimiento informado, aprobado por el Comité de Ética de la Universidad. En todos ellos se determinó peso, estatura, CC y medición de presión arterial (PA), por un equipo previamente capacitado de pediatras y enfermeras. Luego se obtuvo una muestra de sangre con ayuno de 12 horas.

\section{Evaluación clínica}

Se midió peso y estatura mediante balanza de palanca y estadiómetro SECA ${ }^{\circledR}$. Se calculó el IMC, expresado en percentiles. Se definió obesidad como IMC $\geq$ pc95, sobrepeso: pc85-94, eutrofia: pc10-84 y bajo peso $<$ pc10, usando como referencia CDC-NCHS, año $2000^{35}$. Se midió la CC con cinta métrica inextensible, promediándose tres mediciones ${ }^{36}$. Calculamos el ICE, dividiendo la CC por la estatura. Se midió la PA en tres oportunidades, de acuerdo a las normas internacionales, considerándose prehipertensión una PA entre el pc 90 a 94 e hipertensión si la presión arterial sistólica (PAS) y/o diastólica (PAD) eran $\geq$ pc95 para sexo, talla y edad ${ }^{37}$.

\section{Exámenes de sangre}

La glicemia se midió por método de hexoquinasa; colesterol HDL (cHDL) y triglicéridos (TG) por métodos enzimáticos estándar y se calculó el colesterol LDL (cLDL) mediante la fórmula de Friedewald. La PCRus se midió mediante método nefelométrico (Dade Behring). 


\section{Definición de síndrome metabólico}

Se utilizó la definición de Cook y cols ${ }^{6}$ para niños y adolescentes, que considera la presencia de al menos tres de los siguientes componentes: Obesidad abdominal (cintura $\geq$ pc90 del patrón utilizado) (36), cHDL $\leq 40 \mathrm{mg} / \mathrm{dl}, \mathrm{TG} \geq 110 \mathrm{mg} /$ dl (percentiles 25 y 75 para población general norteamericana respectivamente $)^{38}$, glicemia $\geq$ $100 \mathrm{mg} / \mathrm{dl}^{39}$ y PAS y/o PAD $\geq$ Pc90 ${ }^{37}$

\section{Estadística}

Los resultados son expresados como promedio y desviación estándar o intervalo de confianza de 95\%. Las comparaciones de medias provienen de ANOVA de un factor. El SMET fue modelado en función de la ICE y PCRus mediante modelos de regresión logística acumulada bajo el supuesto de odds (chances) proporcionales, verificado mediante prueba de razón de verosimilitud. Este supuesto consiste en que, para los odds en escala logarítmica $(\log (\mathrm{p} /(1-\mathrm{p}))$, donde "p" es la probabilidad de respuesta positiva), se supone sólo una diferencia en intercepto para los distintos niveles de la respuesta, en vez de distintas pendientes, favoreciendo la parsimonia y facilidad de interpretación del modelo. La discriminación entre modelos no anidados se basó en el criterio de información de Akaike (AIC) ${ }^{40}$. La capacidad de discriminación de los mismos fue evaluada a través de la curva ROC. Se utilizó como punto de corte el nivel de la variable explicativa que maximiza de forma conjunta la sensibilidad y especificidad. Todos los cálculos fueron realizados con el paquete estadístico $\mathrm{R}^{41}$, ${ }^{42}$. Se cuantifica el riesgo de presentar "1 o más", "2 o más", o "3 o más" componentes del síndrome metabólico mediante "odds ratio" o "razón de chances" (OR).

\section{Resultados}

En la muestra total, la edad promedio fue de $11,5 \pm 2$ años, con $50 \%$ mujeres y $30 \%$ de prepúberes; según IMC, $53 \%$ de los niños eran eutróficos, $18 \%$ sobrepeso y $29 \%$ obesos. No hubo diferencia en estos grupos según edad, sexo o desarrollo puberal. De los niños obesos, 32 venían del policlínico de niños obesos de la nutrióloga del grupo y 30 niños de la muestra general.

La Tabla 1 muestra los distintos valores promedio, en hombres y mujeres, de los 5 componentes cardiometabólicos del SMET, ICE y PCRus. No se encontraron diferencias significativas entre sexos, salvo en la glicemia, pero en ambos estaba en rangos normales. De acuerdo al criterio de Cook, 5\% del total de niños y 18\% de los obesos cumplieron criterios de SMET. De acuerdo a la agregación de componentes del SMET, 95 niños tenían cero componente, 79 niños un componente, 24 dos componentes y 11, 3 componentes del SMET. Por lo tanto, 11 de 209 tenían SMET y todos ellos eran obesos. En la medida que se iban agregando 1, 2 ó 3 componentes del SMET, aumentaron significativamente los valores de cintura, PAS, PAD, triglicéridos, ICE y PCRus y disminuyó significativamente el CHDL. La glicemia no aumentó significativamente al aumentar el número de componentes del SMET.

En la Tabla 2 se observa el punto de corte óptimo, tanto para ICE como para PCRus, respecto a la presencia o ausencia de SMET y según el número de componentes del SMET. El OR para ICE fue de $1,20(1,15-1,25)$ y el OR para PCRus fue de 1,6 (1,3-2,1). Según el criterio de información de Akaike, el ICE resultó mejor predictor de SMET que la PCRus. De igual forma, esta Tabla muestra los puntos de corte de ICE y PCRus que predicen, con una mayor sensibilidad y especificidad, la agregación de 1 o más, 2 o más y 3 o más componentes cardiometabólicos.

Las Figuras 1 y 2 muestran al ICE y a la PCRus como predictores de riesgo de agregación de componentes del SMET ( 1 o más, 2 o más, 3 o más factores) usando el modelo de odds (chances) proporcionales. Para ICE (Figura 1), el OR de aumentar en un componente del SMET fue de 1,20 $(1,15-1,25)$ por centésima de ICE. Es decir, por cada 0,01 de aumento del ICE, existe un riesgo de $20 \%$ de presentar $1 \mathrm{o}+, 2 \mathrm{o}+, 3 \mathrm{o}+$ componentes del SMET. A modo de ejemplo, un niño con un ICE de 0,42 (es decir, con un valor de ICE menor al punto de corte de 0,55 , que tiene muy buena sensibilidad y especificidad), se observa que hay un $16 \%$ de riesgo de tener $10+$ componentes del SMET, $4 \%$ de tener 2 o + y cerca de $0 \%$ de tener 3 o + componentes. En cambio un niño con ICE de 0,6 (es decir, con un ICE mayor al punto de corte), presenta $84 \%$ de probabilidad de tener 1 o + componentes del SMET, 30\% de tener 2 o + y cerca de $10 \%$ de presentar 3 o + componentes.

Para PCRus, el OR fue de 1,62 (1,26-2,09) (Figura 2). En este caso un niño con una PCRus 
Riesgo cardiometabólico en niños y adolescentes - P. Arnaiz et al

$=0,21 \mathrm{mg} / \mathrm{L}$ (percentil 25 de la muestra), presenta aproximadamente $40 \%$ de probabilidad de presentar $1 \mathrm{o}+$ componentes del SMET, $10 \%$ de $2 \mathrm{o}+$ y cerca de $4 \%$ de $3 \mathrm{o}+$. Por el contrario, un paciente con una PCRus $=0,69 \mathrm{mg} / \mathrm{L}$ (percentil 75 ), presenta aproximadamente $58 \%$ de riesgo de tener $1 \mathrm{o}+$ componentes del SMET, $18 \%$ de tener 2 o + y cerca de $5 \%$ de 3 o más.

Si descartamos el efecto que tiene la cintura sobre los predictores (ICE y PCRus), el OR de aumentar en un componente, distinto al de cir- cunferencia de cintura, por centésima de ICE es de 1,05 (1,01-1,09). En cambio el OR de aumentar en un componente, distinto al de cintura para log PCRus, no resultó estadísticamente significativo (OR 0,98 (0,75-1,28) (Tabla 3). En esta misma Tabla, el punto de corte para predecir SMET, con criterios distintos de la cintura, es de 0,55 , con sensibilidad de $41 \%$ y especificidad de $74 \%$ para 1 o más componentes. Para 2 o más componentes, la sensibilidad baja a $13 \%$ y la especificidad sube a $99 \%$.

Tabla 1. Características antropométricas y componentes del SMET en 209 niños y adolescentes

\begin{tabular}{|lcccc|}
\hline & Hombres $(\mathbf{n}=\mathbf{1 0 5})$ & Mujeres $(\mathbf{n}=\mathbf{1 0 4})$ & Total $(\mathbf{n}=\mathbf{2 0 9})$ & p \\
\hline Edad (años) & $12 \pm 2$ & $12 \pm 2$ & $12 \pm 2$ & NS \\
Talla (cm) & $149 \pm 15$ & $147 \pm 13$ & $148 \pm 14$ & NS \\
\hline Cintura (cm) & $77 \pm 14$. & $79 \pm 15$ & $78 \pm 15$ & NS \\
ICE & $0,52 \pm 0,08$ & $0,53 \pm 0,08$ & $0,53 \pm 0,08$ & NS \\
Log(PCRus) & $-0,54 \pm 1,07$ & $-0,51 \pm 1.07$ & $-0,53 \pm 1,07$ & NS \\
PAS(mmHg) & $106 \pm 9,9$ & $103 \pm 9,7$ & $105 \pm 9,9$ & 0,079 \\
PAD (mmHg) & $56 \pm 5,3$ & $56 \pm 5,5$ & $56 \pm 5,4$ & NS \\
Glucosa (mg/dl) & $86 \pm 5$ & $83 \pm 5$ & $84 \pm 5$ & $<0,001$ \\
\hline CHDL (mg/dl) & $54 \pm 12$ & $52 \pm 11$ & $53 \pm 12$ & NS \\
TG (mg/dl) & $70 \pm 37$ & $74 \pm 36$ & $72 \pm 37$ & NS \\
\hline
\end{tabular}

ICE: índice cintura estatura; LogPCR: logaritmo de PCR ultrasensible, PAS: presión arterial sistólica; PAD: presión arterial diastólica; TG: triglicéridos. Valores expresados en promedio \pm DES

Tabla 2. Odds ratio para ICE y PCRus en el modelo de odds proporcionales para predecir $10+, 20+, 30+$ componentes del síndrome metabólico, con los puntos de cortes que maximizan la sensibilidad y especificidad del ICE y PCRus

\begin{tabular}{|c|c|c|c|c|c|}
\hline & & & SM 1+ & SM 2+ & SM 3+ \\
\hline ICE & & Punto de corte & 0,55 & 0,54 & 0,57 \\
\hline \multirow[t]{3}{*}{$O . R^{*}$. } & $1,20(1,15-1,25)$ & Sensibilidad (\%) & 93,9 & 36 & 16,7 \\
\hline & & Especificidad (\%) & 70,9 & 96,7 & 100 \\
\hline & & $\begin{array}{l}\text { Correctamente } \\
\text { clasificados (\%) }\end{array}$ & 79,9 & 71,8 & 73,7 \\
\hline PCRus & & Puntos de corte & 0,61 & 0,63 & 0,8 \\
\hline \multirow[t]{3}{*}{ O.R. ** } & $1,6 \quad(1,3-2,1)$ & Sensibilidad (\%) & 69 & 23,4 & 8,6 \\
\hline & & Especificidad (\%) & 59,8 & 88,5 & 96,9 \\
\hline & & Correctamente clasificado (\%) & 63,6 & 58,4 & 62,2 \\
\hline
\end{tabular}

*Correspondiente a 100*ICE. **Correspondiente Log PCRus. ICE: índice cintura estatura. PCRus: Proteína C-reactiva ultrasensible. En la medición de los puntos de cortes de PCRus, estos están expresados en mg/L. 


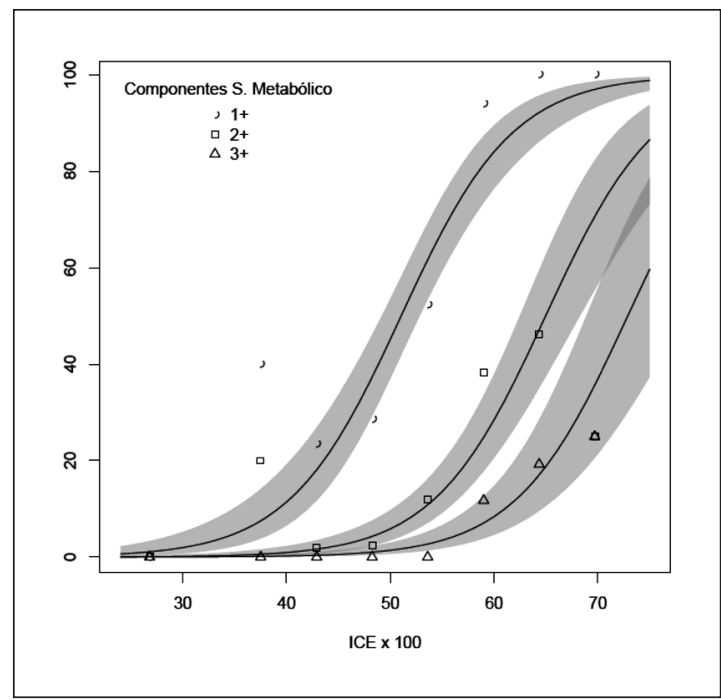

Figura 1. Riesgo en porcentaje de presentar $10+, 20+$, 3 o + factores de riesgo cardiometabólicos del síndrome metabólico, de acuerdo a los valores de índice cintura estatura.

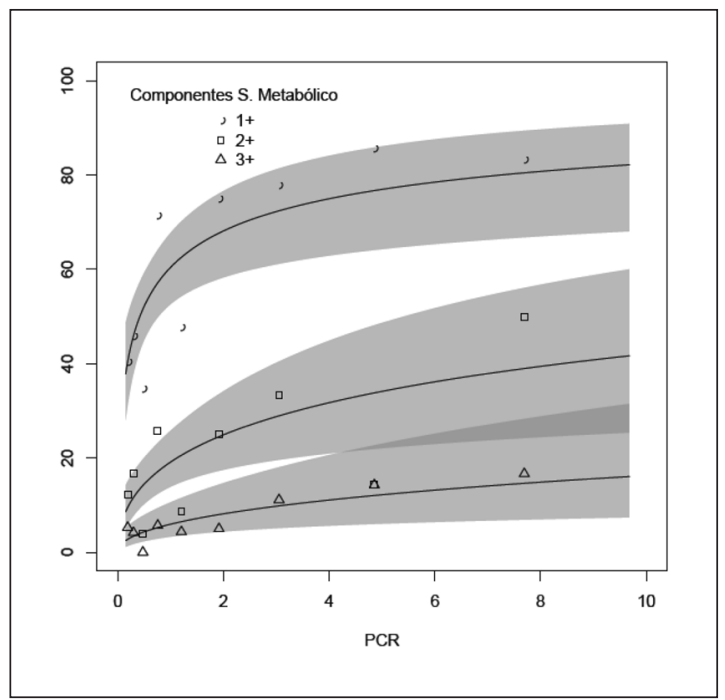

Figura 2. Riesgo en porcentaje de presentar $10+, 20+$, 3 o + factores de riesgo cardiometabólicos del síndrome metabólico, de acuerdo a los valores de Proteína C-reactiva ultrasensible.

Tabla 3. Odds ratio para ICE y PCRus en el modelo de odds proporcionales para predecir 10 + y 20 + componentes del síndrome metabólico, excluyendo circunferencia de cintura, con los puntos de cortes que maximizan la sensibilidad y especificidad del ICE y PCRus

\begin{tabular}{|c|c|c|c|c|}
\hline & & & SM 1+ & SM 2+ \\
\hline ICE & & Punto de corte & 0,55 & 0,55 \\
\hline \multirow[t]{3}{*}{$O \cdot R^{*}$. } & $1,05(1,01-1,09)$ & Sensibilidad (\%) & $41 \%$ & $12,8 \%$ \\
\hline & & Especificidad (\%) & $74 \%$ & $99,2 \%$ \\
\hline & & Correctamente clasificados (\%) & $61,7 \%$ & $67 \%$ \\
\hline \multicolumn{5}{|l|}{ PCRus } \\
\hline O.R. ${ }^{* *}$ & $0,98(0,75-1,28)$ & & & \\
\hline
\end{tabular}

*Correspondiente a $100 *$ ICE. ${ }^{*}$ Correspondiente Log PCRus. ICE: índice cintura estatura. PCRus: Proteína C-reactiva ultrasensible.

Debemos destacar que el propósito del modelo estadístico aplicado, es la estimación del odds ratio o chance de aumentar en un componente del SMET, y no, hacer inferencia con respecto a la prevalencia de los componentes del SMET en la población.

\section{Discusión}

Este estudio demuestra que ambos, el ICE y la PCRus predicen el riesgo de "agregación" de componentes cardiometabólicos en niños y adolescentes de Santiago, siendo el ICE más sencillo de aplicar y mejor predictor estadístico en este grupo. Nuestros resultados enfatizan el concepto de que "la suma de componentes" es lo importante desde el punto de vista predictivo, más que la definición de "sí-no" que se ha aplicado para la definición actual de SMET. En niños, esta visión de riesgo es fundamental ya que la mayoría presenta menos de 3 componentes clásicos.

La obesidad iniciada en la niñez, el sedentarismo reinante entre los niños y jóvenes, sumado a los 
antecedentes familiares de riesgo cardiovascular, conducen a la instalación progresiva de SMET durante la edad pediátrica. Los niños con una sumatoria de componentes cardiometabólicos constituyen grupos de mayor riesgo. Estos tendrían un inicio temprano de la ateroesclerosis y una evolución más rápida de la misma hacia la edad adulta, desarrollando, tempranamente en la vida, enfermedad cardiovascular y diabetes tipo $2^{4,5}$. Por esto, es de gran utilidad contar con parámetros bioquímicos, antropométricos y/o de imágenes predictores de riesgo cardiovascular o metabólico futuros, y aplicables en niños y adolescentes para poder desarrollar puntos de corte sencillos de aplicar en la práctica clínica ${ }^{43,44}$. En la actualidad, especialmente en niños y adolescentes que están en desarrollo, se piensa que no existe un umbral sobre el cual ya no se esté saludable y bajo el cual se goce de buena salud. Existe una relación continua entre la agregación de factores de riesgo y las alteraciones que estos van causando. Este enfoque es novedoso y no existen actualmente estudios en niños que lo enfaticen. Actualmente, los índices antropométricos más usados como predictores de riesgo para desarrollar SMET y resistencia a la insulina en niños y adolescentes son el IMC y en menor medida la medición de la CC y el ICE. Estos últimos parámetros se están usando cada vez más en clínica, con la idea de prevenir la progresión del riesgo cardiovascular inicial y de esta manera poder desarrollar programas de intervención y seguimiento en edades tempranas de la vida. A su vez, estos parámetros serían más sensibles a la etnia, sexo, edad y pubertad.

Nuestro estudio demuestra que el ICE y la PCRus son buenos predictores de "agregación" de componentes del SMET, siendo el ICE más sencillo y barato de aplicar y mejor predictor estadístico que la PCRus.

Sin duda, una crítica a nuestro modelo es que el ICE contempla la cintura dentro del cálculo del índice cintura/estatura y sabemos que la cintura es un componente del SMET. Por ello también quisimos desarrollar un modelo que excluyera la cintura dentro de los componentes del SMET. Así se ajustó un modelo para la probabilidad de tener $1 \mathrm{o}+\mathrm{o} 2 \mathrm{o}+$ componentes, con los 4 componentes distintos de la cintura. En este último caso, el ICE también resultó significativo para predecir riesgo de agregación de componentes del SMET (Tabla 3).
Finalmente, nuestros resultados enfatizan el concepto de que es la "sumatoria de componentes cardiometabólicos" lo más importante desde el punto de vista predictivo, más que la definición absoluta "sí-no" de presencia o ausencia de SMET. En las Figuras 1 y 2 mostramos que tanto para el ICE como para la PCRus, el que un niño tenga un solo componente o 2 y no llegue a tener todavía el SMET propiamente tal (más de 3 componentes), no descarta que ese niño pueda desarrollarlo en el futuro. Desde el punto de vista de salud pública, esta visión del riesgo es fundamental, ya que la mayoría de los niños presenta menos de 3 componentes del SMET.

De esta manera el ICE puede ser considerado un marcador "al lado de la cama del paciente", siendo fácil de aplicar en la consulta ambulatoria, sencillo de calcular sin necesidad de tablas y percentiles y permite hacer un seguimiento individual a lo largo de los sucesivos controles de salud.

Estudios recientes de la cohorte de Bogalusa han demostrado la capacidad predictiva del ICE, proponiéndolo como un buen predictor de riesgo cardiovascular ${ }^{30}$.

Nuestro estudio tiene limitaciones: es un estudio de corte transversal, lo cual supone una capacidad limitada en la estimación de riesgo, en nuestro caso de componentes del SMET, lo cual hace que nuestros resultados se encuentren en la categoría de asociaciones y no de causalidad. De igual forma nuestra muestra es pequeña y la edad está en un rango estrecho $12 \pm 2$ años, lo cual limita la aplicación de nuestros resultados. Así mismo, la población estudiada no fue estrictamente homogénea ya que incluimos población escolar general no consultante y otra población portadora de sobrepeso y obesidad que consultó a una especialista.

\section{Referencias}

1. JUNAEB. Situación nutricional de los escolares de primero básico 2008. Disponible en: htpp://sistemas. JUNAEB.cl/estadosnutricionales2008/index2.php. 2009.

2. INE. Instituto Nacional de Estadísticas de Chile. Disponible en: http://www.INE.cl/canales/chile_estadistico/ demografia_y_vitales/estadisticas_vitales/estadisticas_vitales.php. 2008.

3. Gami AS, Witt BJ, Howard DE, Erwin PJ, Gami LA, Somers VK, et al. Metabolic syndrome and risk of incident 
cardiovascular events and death: A systematic review and meta-analysis of longitudinal studies. J Am Coll Cardiol 2007; 49: 403-14.

4. Morrison JA, Friedman LA, Gray-McGuire C. Metabolic syndrome in childhood predicts adult cardiovascular disease 25 years later: The Princeton lipid research clinics follow-up study. Pediatrics 2007; 120: 340-5.

5. Morrison JA, Friedman LA, Wang P, Glueck CJ. Metabolic syndrome in childhood predicts adult metabolic syndrome and type 2 diabetes mellitus 25 to 30 years later. J Pediatr 2008; 152: 201-6.

6. Cook S, Weitzman M, Auinger P, Nguyen M, Dietz WH. Prevalence of a metabolic syndrome phenotype in adolescents: Findings from the third national health and nutrition examination survey, 1988-1994. Arch Pediatr Adolesc Med 2003; 157: 821-7.

7. de Ferranti SD, Gauvreau K, Ludwig DS, Neufeld EJ, Newburger JW, Rifai N. Prevalence of the metabolic syndrome in American adolescents: Findings from the third national health and nutrition examination survey. Circulation 2004; 110: 2494-7.

8. Weiss R, Dziura J, Burgert TS, Tamborlane WV, Taksali SE, Yeckel CW, et al. Obesity and the metabolic syndrome in children and adolescents. N Engl J Med 2004; 350: 2362-74.

9. Barja S, Arteaga A, Acosta AM, Hodgson MI. [Insulin resistance and other expressions of metabolic syndrome in obese Chilean children]. Rev Med Chile 2003; 131: 259-68.

10. Burrows R, Burgueno M, Leiva L, Ceballos X, Guillier I, Gattas V, et al. [Cardiovascular risk and metabolic profile in obese children and adolescents with low insulin sensitivity]. Rev Med Chile 2005; 133: 795-804.

11. World Health Organization. Obesity: Preventing and managing the global epidemic. Report of a WHO consultation on obesity. 1998.

12. Clinical guidelines on the identification, evaluation, and treatment of overweight and obesity in adults-the evidence report. National institutes of health. Obes Res 1998; 6 Suppl 2: 51S-209S.

13. Wang Y, Rimm EB, Stampfer MJ, Willett WC, Hu FB. Comparison of abdominal adiposity and overall obesity in predicting risk of type 2 diabetes among men. Am J Clin Nutr 2005; 81: 555-63.

14. Katzmarzyk PT, Craig CL. Independent effects of waist circumference and physical activity on all-cause mortality in Canadian women. Appl Physiol Nutr Metab 2006; 31:271-6.

15. Yusuf S, Hawken S, Ounpuu S, Bautista L, Franzosi MG, Commerford $\mathrm{P}$, et al. Obesity and the risk of myocardial infarction in 27,000 participants from 52 countries: A case-control study. Lancet 2005; 366: 1640-9.

16. Hu G, Tuomilehto J, Silventoinen K, Barengo N, Jousilahti P. Joint effects of physical activity, body mass index, waist circumference and waist-to-hip ratio with the risk of cardiovascular disease among middle-aged Finish men and women. Eur Heart J 2004; 25: 2212-9.

17. Lofgren I, Herron K, Zern T, West K, Patalay M, Shachter NS, et al. Waist circumference is a better predictor than body mass index of coronary heart disease risk in overweight premenopausal women. J Nutr 2004; 134: 1071-6.

18. Carey VJ, Walters EE, Colditz GA, Solomon CG, Willett WC, Rosner BA, et al. Body fat distribution and risk of non-insulin-dependent diabetes mellitus in women. The nurses' health study. Am J Epidemiol 1997; 145: 614-9.

19. Visscher TL, Seidell JC, Molarius A, van der Kuip D, Hofman A, Witteman JC. A comparison of body mass index, waist-hip ratio and waist circumference as predictors of all-cause mortality among the elderly: The Rotterdam study. Int J Obes Relat Metab Disord 2001; 25: $1730-5$.

20. Katzmarzyk PT, Srinivasan SR, Chen W, Malina RM, Bouchard C, Berenson GS. Body mass index, waist circumference, and clustering of cardiovascular disease risk factors in a biracial sample of children and adolescents. Pediatrics 2004; 114: e198-205.

21. Lee S, Bacha F, Gungor N, Arslanian SA. Waist circumference is an independent predictor of insulin resistance in black and white youths. J Pediatr 2006; 148: 188-94.

22. Hirschler V, Aranda C, Calcagno M de L, Maccalini G, Jadzinsky M. Can waist circumference identify children with the metabolic syndrome? Arch Pediatr Adolesc Med 2005; 159: 740-4.

23. Caballero AE, Bousquet-Santos K, Robles-Osorio L, Montagnani V, Soodini G, Porramatikul S, et al. Overweight Latino children and adolescents have marked endothelial dysfunction and subclinical vascular inflammation in association with excess body fat and insulin resistance. Diabetes Care 2008; 31: 576-82.

24. Lee CM, Huxley RR, Wildman RP, Woodward M. Indices of abdominal obesity are better discriminators of cardiovascular risk factors than BMI: A meta-analysis. J Clin Epidemiol 2008; 61: 646-53.

25. Gelber RP, Gaziano JM, Orav EJ, Manson JE, Buring JE, Kurth T. Measures of obesity and cardiovascular risk among men and women. J Am Coll Cardiol 2008; 52: 605-15.

26. Freedman DS, Kahn HS, Mei Z, Grummer-Strawn LM, Dietz WH, Srinivasan SR, et al Relation of body mass index and waist-to-height ratio to cardiovascular disease risk factors in children and adolescents: The Bogalusa 
heart study. Am J Clin Nutr 2007; 86: 33-40.

27. Maffeis C, Banzato C, Talamini G. Waist-to-height ratio, a useful index to identify high metabolic risk in overweight children. J Pediatr 2008; 152: 207-13.

28. Savva SC, Tornaritis M, Savva ME, Kourides Y, Panagi A, Silikiotou N, et al. Waist circumference and waistto-height ratio are better predictors of cardiovascular disease risk factors in children than body mass index. Int J Obes Relat Metab Disord 2000; 24: 1453-8.

29. Kahn HS, Imperatore G, Cheng YJ. A population-based comparison of BMI percentiles and waist-to-height ratio for identifying cardiovascular risk in youth. J Pediatr 2005; 146: 482-8

30. Freedman DS, Dietz WH, Srinivasan SR, Berenson GS. Risk factors and adult body mass index among overweight children: The Bogalusa heart study. Pediatrics 2009; 123: 750-7.

31. Koch E, Díaz C, Romero T, Kirschbaum A, Manríquez L, Paredes M. Razón cintura-estatura como un predictor de mortalidad en población chilena: Un estudio de 8 años de seguimiento en la cohorte del proyecto San Francisco. Rev Chil Cardiol 2007; 26: 145-50.

32. Ross R. Atherosclerosis--an inflammatory disease. N Engl J Med 1999; 340: 115-26.

33. Lagrand WK, Visser CA, Hermens WT, Niessen HW, Verheugt FW, Wolbink GJ, et al. C-reactive protein as a cardiovascular risk factor: More than an epiphenomenon? Circulation 1999; 100: 96-102.

34. Acevedo M, Arnaiz P, Barja S, Bambs C, Berríos X, Guzmán B, et al. Relationship of c-reactive protein to adiposity, cardiovascular risk factors and subclinical atherosclerosis in healthy children. Rev Esp Cardiol 2007; 60: 1051-8.

35. CDC. Center for Disease Control (CDC) growth charts. http://www.CDC.gov/growthcharts.
36. Fernández JR, Redden DT, Pietrobelli A, Allison DB. Waist circumference percentiles in nationally representative samples of African American, European American, and Mexican American children and adolescents. J Pediatr 2004; 145: 439-44.

37. The fourth report on the diagnosis, evaluation, and treatment of high blood pressure in children and adolescents. Pediatrics 2004; 114: 555-76.

38. NCEP. National cholesterol education program report of the expert panel on blood cholesterol levels in children and adolescents. National heart and blood institute information center. 1991.

39. Gabir MM, Hanson RL, Dabelea D, Imperatore G, Roumain J, Bennett PH, et al. The 1997 American diabetes association and 1999 world health organization criteria for hyperglycemia in the diagnosis and prediction of diabetes. Diabetes Care 2000; 23: 1108-12.

40. Akaike H. A new look at the statistical model identification. IEEE Transactions on Automatic Control 1974; 19: 716-23.

41. R development core team. R: A language and environment for statistical computing. $\mathrm{R}$ foundation for statistical computing, Vienna, Austria. http://www.R-project. org. 2009.

42. Wood SN. Fast stable direct fitting and smoothness selection for generalized additive models. Journal of the Royal Statistical Society: Series B 2008; 70: 495-518.

43. Arnaiz P, Acevedo M, Barja S, Aglony M, Guzmán B, Cassis B, et al. Adiponectin levels, cardiometabolic risk factors and markers of subclinical atherosclerosis in children. Int J Cardiol 2010; 138: 138-44.

44. Arnaiz P, Acevedo M, Barja S, Berríos X, Guzmán B, Bambs $C$, et al. Arteriosesclerosis subclínica, factores de riesgo cardiovascular clásicos y emergentes en niños obesos chilenos. Rev Chil Ped 2007; 78: 135-42. 